\title{
Correction: Development of a highly controlled system for large-area, directional printing of quasi-1D nanomaterials
}

\author{
Adamos Christou (D, Fengyuan Liu and Ravinder Dahiya (1)
}

Correction to: Microsystems \& Nanoengineering https://doi.org/10.1038/s41378-021-00314-6

published online 19 October 2021

Following publication of this article ${ }^{1}$, it is reported the Acknowledgement section need to be updated as below: This work is partly supported by the EPSRC through an Engineering Fellowship (EP/R029644/1) and Programme Grant-Heteroprint (EP/R03480X/1) and North West Centre for Advanced Manufacturing project funded by the European Union's INTERREG programme (H2020-Intereg-IVA5055), managed by the Special EU
Programmes Body. The views and opinions in this document do not necessarily reflect those of the European Commission or the SEUPB.

The original article has been updated.

Published online: 26 November 2021

\section{Reference}

1. Christou, A., Liu, F. \& Dahiya, R. Development of a highly controlled system for large-area, directional printing of quasi-1D nanomaterials. Microsyst. Nanoeng. 7, 82, https://doi.org/10.1038/s41378-021-00314-6 (2021). 